\title{
Reducing co-administration of proton pump inhibitors and antibiotics using a computerized order entry alert and prospective audit and feedback
}

\author{
Christopher E. Kandel ${ }^{1}$, Suzanne Gill ${ }^{2}$, Janine McCready ${ }^{3}$, John Matelski ${ }^{4}$ and Jeff E. Powis ${ }^{3^{*}}$
}

\begin{abstract}
Background: Antibiotics and proton pump inhibitors (PPIs) are associated with Clostridium difficile infection (CDI). Both a computer order entry alert to highlight this association as well as antimicrobial stewardship directed prospective audit and feedback represent novel interventions to reduce the co-administration of antibiotics and PPIs among hospitalized patients.
\end{abstract}

Methods: Consecutive patients admitted to two General Internal Medicine wards from October 1, 2010 until March 31, 2013 at a teaching hospital in Toronto, Ontario, Canada were evaluated. The baseline observation period was followed by the first phase, which involved the creation of a computerized order entry alert that was triggered when either a PPI or an antibiotic was ordered in the presence of the other. The second phase consisted of the introduction of an antibiotic stewardship-initiated prospective audit and feedback strategy. The primary outcome was the co-administration of antibiotics and PPIs during each phase.

Results: This alert led to a significant reduction in the co-administration of antibiotics and PPIs adjusted for month and secular trends, expressed as days of therapy per 100 patient days (4.99 vs. 3.14, $p<0.001)$ The subsequent introduction of the antibiotic stewardship program further reduced the co-administration (3.14 vs. 1.80, $p<0.001$ ). No change was observed in adjusted monthly CDI rates per 100 patient care days between the baseline and alert cohorts ( 0.12 vs. $0.12, p=0.99)$ or the baseline and antibiotic stewardship phases $(0.12$ vs. $0.13, p=0.97)$.

Conclusions: Decreasing the co-administration of PPIs and antibiotics can be achieved using a simple automatic alert followed by prospective audit and feedback.

Keywords: Computerized alerts, Antibiotic stewardship, Proton pump inhibitors, Clostridium difficile infection, Prospective audit and feedback

\section{Background}

Hospitalization represents an opportunity to assess medication appropriateness. Proton pump inhibitors (PPIs) are frequently inappropriately used and represent an ideal target for re-evaluation [1, 2]. PPIs increase the risk of Clostridium difficile infection (CDI), including both incident episodes as well as recurrences, by approximately $60 \%[3,4]$. This elevated risk exists irrespective of

\footnotetext{
* Correspondence: jpowi@tegh.on.ca

${ }^{3}$ Division of Infectious Diseases, Toronto East General Hospital, 825 Coxwell

Avenue, Toronto, ON M4C 3E7, Canada

Full list of author information is available at the end of the article
}

whether the infection was acquired in a community or hospital setting $[4,5]$. Antibiotics also increase the risk of nosocomial CDI, particularly when prescribed concurrently with a PPI [6-9]. Accordingly, interventions targeting a reduction in the co-administration of PPIs and antibiotics may be beneficial.

Automated alerts activated at the time of computer order entry (CPOE) have been used to reduce medical errors through warnings of impending drug-drug interactions and unrecognized drug allergies. Similar interventions have successfully altered PPI prescribing patterns in the hospital setting and changed antibiotic 
prescribing patterns $[10,11]$. A similar automated strategy may reduce concurrent PPI and antibiotic use, but this has not yet been tested. Extending the purview of antibiotic stewardship programs (ASP), which are successful in lowering antibiotic use in the acute care setting, to identify medications with the potential to increase the harms of antibiotics is another potential intervention to limit co-administration [12].

We evaluated the impact of a real-time automated computer physician order entry (CPOE) alert followed by an ASP-initiated prospective audit and feedback (PAF) strategy on rates of co-administration of PPI's and antibiotics in a large teaching hospital in Ontario, Canada.

\section{Methods}

\section{Study design}

We prospectively evaluated consecutive patients admitted to two General Internal Medicine wards from October 1, 2010 until March 31, 2013 at a 490-bed teaching hospital in Toronto, Ontario, Canada. The baseline observation interval occurred from October 1, 2010 until September 30, 2011. The first phase, from October 1, 2011 until March 31, 2012, involved the implementation of a CPOE alert that was triggered when either a PPI or an antibiotic was ordered in the presence of the other. This alert highlighted the association of concurrent PPI's and antibiotics with the increased risk of subsequent CDI. Embedded within the alert was an educational tool that assisted with a risk-benefit analysis of whether to stop a PPI, which for example should be continued in the setting of a recent upper gastrointestinal bleed. The second phase consisted of the introduction of an ASPinitiated PAF strategy, which occurred from April 1, 2012 until March 31, 2013. PAF was triggered from Monday to Friday when an inpatient was prescribed a systemic antimicrobial agent. The aim of the ASP was to provide recommendations regarding the appropriateness of antimicrobial therapy as well as to note the concomitant use of a PPI. Ethics approval was granted from Toronto East General Hospital's Research Ethics Board.

\section{Outcome measures}

Inpatient orders of antibiotics and PPIs were obtained from the computer order entry system (PowerChart, Cerner Canada, Markham, Canada) and reported as days of therapy (DOT) for each individual medication per 100 inpatient days. One DOT represents the administration of a single medication on a given day irrespective of dosing frequency or strength. Hospital-acquired CDI was confirmed using the Ontario Ministry of Health and Long Term Care case definition with toxin detection by enzyme immunoassay or polymerase chain reaction and presented as a rate over 100 inpatient days [13]. Only loose stool specimens were processed and repeat testing of duplicate specimens within seven days was not permitted. The primary outcome was co-administration of PPI's and antibiotics. Pre-specified secondary outcomes consisted of CDI rates, PPI use, antibiotic use, and the frequency with which the alert was activated. Only the initial occurrence of the triggered alert for each patient during each hospitalization was used for analysis. Countervailing measures included rates of Gastroenterology consultation and receipt of packed red blood cell transfusion. Transfusions are a marker of acute gastrointestinal bleeding while specialist consultation serves to highlight the possible need for guidance in treating conditions exacerbated by the abrupt cessation of PPI's.

\section{Statistical analysis}

Generalized linear models were used to analyze systematic differences between the three intervention periods while adjusting for seasonality (monthly effects) and secular trends. In particular, logistic regression was used for the binary outcomes (co-administration, antibiotics, and PPIs), while Poisson regression was used for the count-based outcomes (rates of CDI, packed red blood cell transfusions, and Gastroenterology consultations). For each outcome, we used the Box-Pierce $P$-value against the null hypothesis that the errors from the adjusted model are uncorrelated. Further, we report the adjusted outcome rate for each period (standardized to events per 100 patient care days), and the Wald Test $P$-value against the null hypothesis of 'no systematic difference' for each pair of time periods (Baseline-Alert, Baseline-Stewardship, and Alert-Stewardship). For the demographic information collected for each cohort we used a one-way ANOVA and a two-tailed chi-square test to assess for differences between continuous and categorical variables, respectively. The analysis was conducted using $\mathrm{R}$ version 3.0.2.

\section{Results}

A total of 54,005 inpatient days were included (21,542 baseline, 10,763 CPOE, and 21,700 ASP). The three cohorts had similar baseline characteristics and discharge diagnoses, aside from a decrease in the proportion of patients with a respiratory illness among the ASP cohort (Table 1). The unadjusted average monthly coadministration of PPI's and antibiotics expressed as DOT per 100 patient days varied between the baseline (5.76, $95 \%$ confidence interval 4.78-6.73), alert (3.47, 2.83-4.11), and ASP cohorts (2.22, 1.75-2.69) (Fig. 1). When adjusting for time and month there was a statistically significant decline in the co-administration of PPIs and antibiotics between the baseline and alert phases (4.99 vs. 3.14 DOT per 100 patient days, $p<0.001$ ), the baseline and ASP phases (4.99 vs. $1.80, p<0.001)$ and 
Table 1 Baseline demographic characteristics for each study cohort

\begin{tabular}{|c|c|c|c|c|}
\hline Variable & Baseline & Computer alert & Antibiotic stewardship & $p$ value \\
\hline Age (mean years $\pm S D$ ) & $71.22 \pm 17.64$ & $71.10 \pm 17.13$ & $72.47 \pm 16.67$ & 0.0212 \\
\hline Male sex (\%) & 45.92 & 44.36 & 45.71 & 0.6706 \\
\hline Patient care days (mean \pm SD) & $1795.17 \pm 116.64$ & $1793.83 \pm 92.16$ & $1808.33 \pm 115.74$ & 0.9513 \\
\hline \multicolumn{5}{|l|}{ Diagnosis at hospital discharge } \\
\hline Blood \& lymphatic system & $56(2)$ & $28(2)$ & $51(2)$ & 0.9324 \\
\hline Circulatory system & $216(9)$ & $119(10)$ & $179(8)$ & 0.0581 \\
\hline Digestive system & $310(14)$ & $175(15)$ & $299(13)$ & 0.281 \\
\hline Ear, nose, mouth \& throat & $21(1)$ & $16(1)$ & $24(1)$ & 0.4354 \\
\hline Endocrine system, nutrition \& metabolism & $143(6)$ & $66(6)$ & $115(5)$ & 0.2956 \\
\hline Hepatobiliary system \& pancreas & $171(7)$ & $95(8)$ & $182(8)$ & 0.5827 \\
\hline Kidney, urinary tract \& male reproductive system & $218(10)$ & $107(9)$ & $185(8)$ & 0.3295 \\
\hline Musculoskeletal system \& connective tissue & $103(5)$ & $66(6)$ & $114(5)$ & 0.2569 \\
\hline Nervous system & $234(10)$ & $123(11)$ & $276(12)$ & 0.0588 \\
\hline Respiratory system & $187(8)$ & $75(7)$ & $89(4)$ & $<.0001$ \\
\hline Skin, subcutaneous tissue $\&$ breast & $82(4)$ & $41(4)$ & $82(4)$ & 0.9803 \\
\hline Multisystem or unspecified site infections & $62(3)$ & $26(2)$ & $78(4)$ & 0.0951 \\
\hline Other & $486(21)$ & $206(18)$ & $551(25)$ & $<.0001$ \\
\hline
\end{tabular}

Unless otherwise specified, data are no. (\%) of patients

Abbreviations: SD standard deviation

Other includes burns, diseases of the eye; female reproductive system; mental diseases \& disorders; miscellaneous \& ungroupable data; other reasons for hospitalization; pregnancy \& childbirth; significant trauma, injury, poisoning \& toxic effects of drugs

the alert and ASP (3.14 vs. $1.80, p<0.001)$. Antibiotic administration significantly declined over each successive time period from the baseline to the alert and through the initiation of the ASP (18.98 DOT per 100 inpatient days, 14.90 , and 8.90 , respectively), which was mirrored by a decline in PPIs $(33.52,29.50$, and 23.95, respectively) (Fig. 2).

The average monthly frequency of the triggered alert was 25.5 during the alert cohort and $16.9(p=0.002)$ during the ASP cohort (Fig. 3). Adjusted monthly CDI rates per 100 inpatient days did not differ between the baseline and alert phases $(0.12$ vs. $0.12, p=0.99)$, the baseline and ASP $(0.12$ vs. $0.13, p=0.97)$ or between the alert and ASP (0.12 vs. 0.13, $p=0.95)$. Similarly, no changes in adjusted monthly packed red blood cell transfusion rates $(1.30$ vs. $1.36, p=0.80)$ or Gastroenterology consultations ( 2.69 vs. $2.96, p=0.42$ ) between the baseline and alert groups and between the baseline and ASP cohorts ( 1.30 vs. $1.18, p=0.71$ and 2.69 vs. 2.16 , $p=0.24$, respectively) were observed.

\section{Discussion}

We observed a reduction in antibiotic and PPI co-administration following the initiation of both an automated computer order entry alert as well as the introduction of ASP initiated PAF. The alert led to a decrease in PPIs and antibiotics that was immediate and simple to implement. The integration of PPI optimization to the responsibilities of an ASP afforded further reductions in co-administration beyond that realized from the alert and allowed for sustainability of the effect.

Automated alerts can alter PPI prescribing patterns and may have prompted clinicians to reconsider the utility of PPI's in the high-risk setting of prescribing antibiotics $[6,10]$. The alert may have engendered an additional evaluation of the risk-benefit ratio of prescribing antibiotics that cumulatively led to the reduction in antibiotic use observed. Lowering overall antibiotic use has been associated with a reduced incidence of nosocomial CDI while it is unclear whether a similar benefit occurs with PPIs [14]. The decrease in co-administration was not associated with a reduction in CDI rates, however the low overall event rate limited statistical power. Antibiotic classes variably affect CDI risk yet in the hospital setting cumulative antibiotic exposure is an independent risk factor for CDI making it imperative to target all antibiotics and not restrict the alert to only those associated with an elevated risk $[15,16]$. The abrupt cessation of a PPI results in rebound acid hypersecretion that can potentially exert a protective effect against contracting $C$. difficile through an increase in gastric acidity [17]. The reduction in PPIs did not result in a change in the rates of red blood cell transfusion or Gasteroenterology consultation, both of which are relatively crude markers of harm 


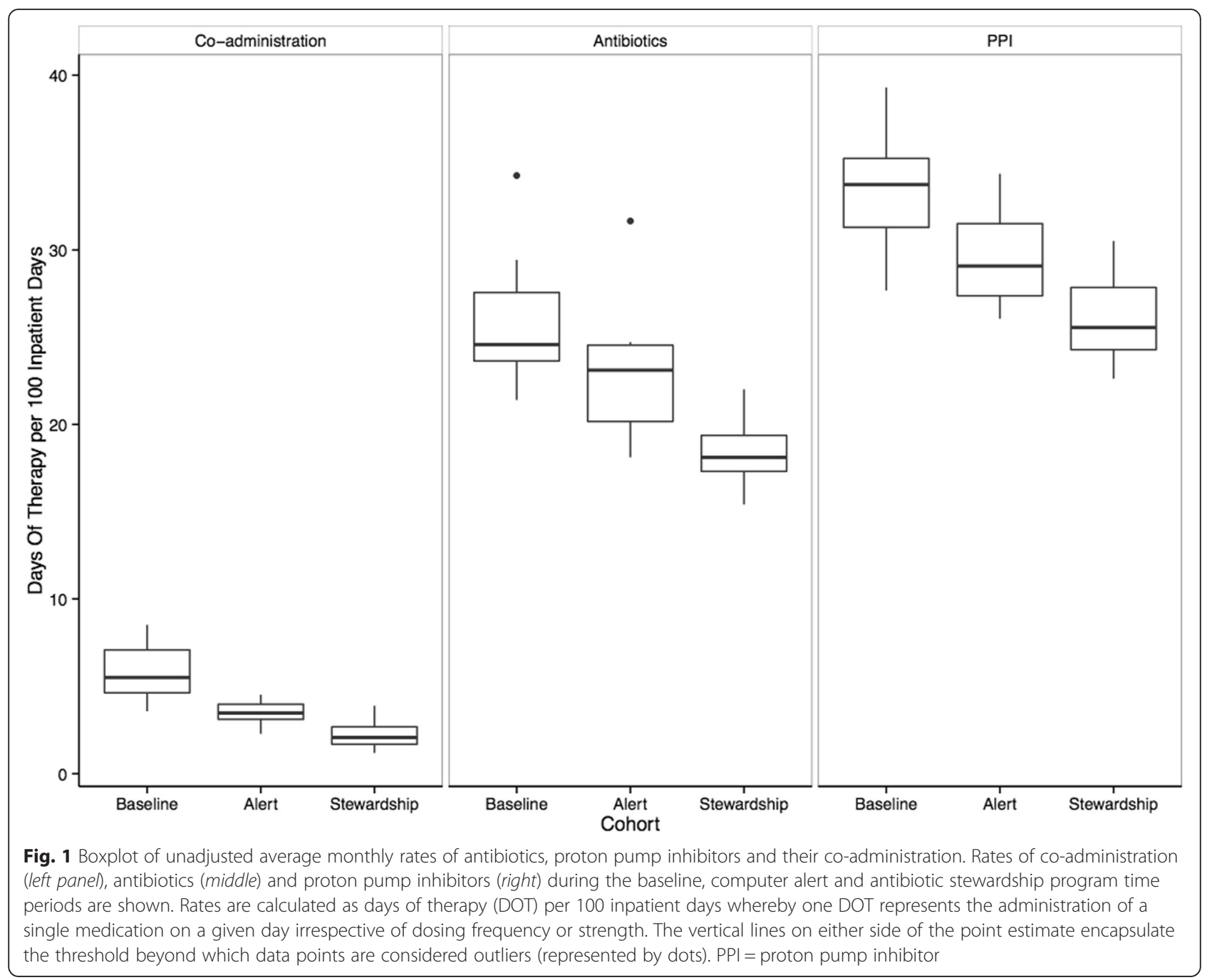

The introduction of PAF by the ASP was followed by further reduction in antibiotic and PPI co-administration compared to both the baseline and the alert cohorts. The reduction in antibiotic administration is commensurate with the effectiveness of ASPs in the inpatient setting [12]. Additionally, the ASP led to a reduction in PPI administration, suggesting that it is feasible to expand the purview of ASPs beyond solely antimicrobials. The decrease in alert frequency following the introduction of the ASP suggests a persistent effect on prescribing practices and cumulative benefit through enacting culture change mediated by the ASP. Importantly, the ASP led to a sustained reduction in the co-administration of PPIs and antibiotics for an additional 12 months, obviating the loss of motivation to follow alert recommendations commonly responsible for the decreased effectiveness of

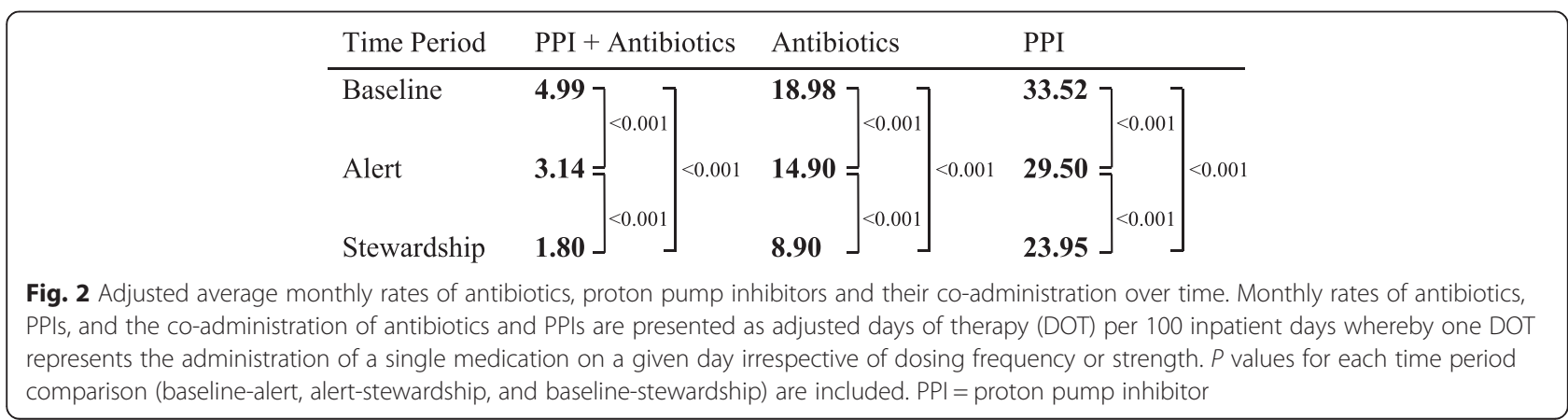




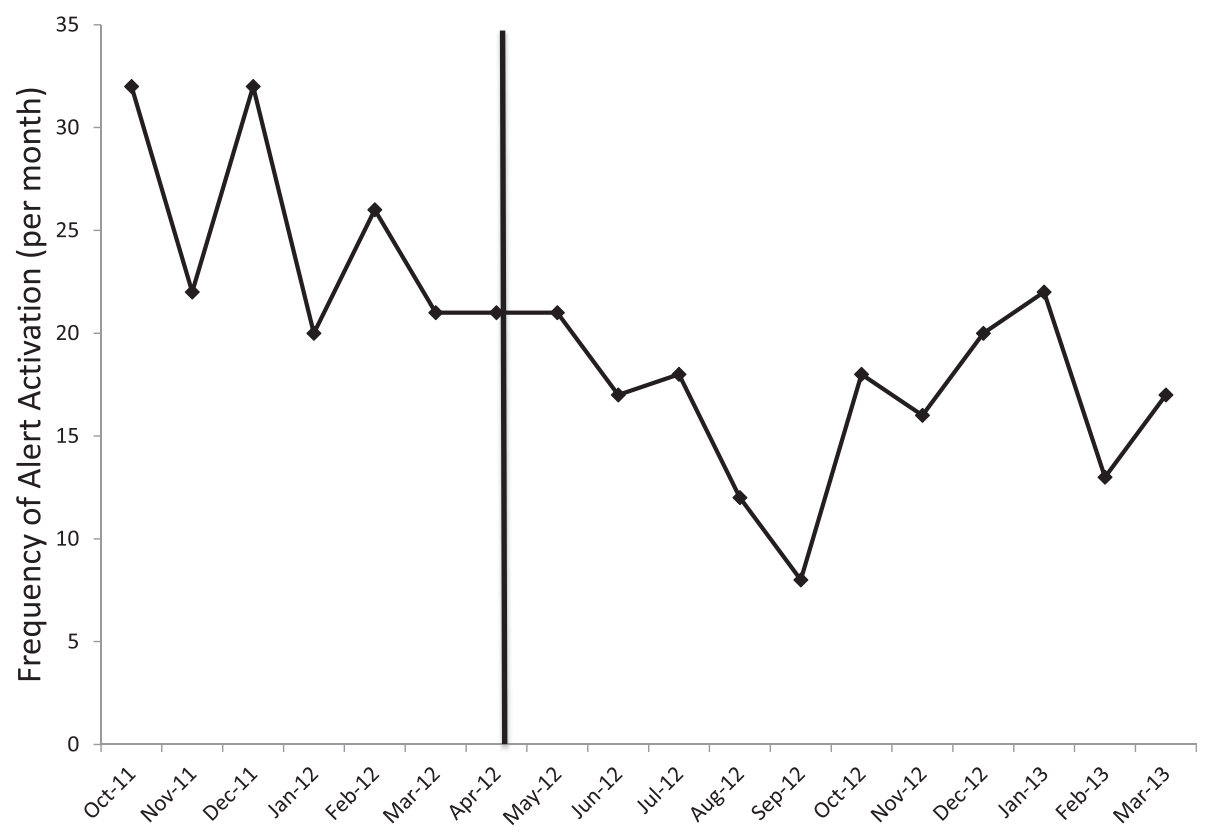

Fig. 3 Frequency of computer order entry alert activation. Number of events per month the alert was activated, which occurred when a proton pump inhibitor or antibiotic was ordered in the presence of the other before and after the implementation of the antibiotic stewardship program (depicted by the vertical line). Only the first instance the alert was triggered for each patient during each hospitalization was included

electronic alerts that occurs over time [18]. ASPs should evolve to encompass more than assessing antibiotic appropriateness, as they are ideally situated to provide advice towards attenuating the potential harms of antibiotics, such as decreasing PPI and antibiotic co-administration.

There are some limitations that merit emphasis. Firstly, the short duration of observation may not accurately reflect the potential benefits observed. Although we adjusted for seasonality in our analysis, the total duration of the study was short and the CDI event rate was low. The low CDI event rate also precluded analyzing risk factors for CDI, including patient-related characteristics such as age and underlying comorbidities, which may have contributed to the absence of statistically significant differences in CDI rates [19]. Secondly, the single site and pre-existing comprehensive ASP, which is activated upon the prescribing of an antibiotic for all inpatients, may affect external validity. Thirdly, robust measures of harm could not be collected and may be underappreciated, especially in light of the short study interval. Fourthly, outcomes were restricted to inhospital events, which may underestimate the benefit of the interventions.

\section{Conclusions}

Reducing the co-administration of PPI's and antibiotics can be achieved with an automatic computerized alert and implementation of an ASP. The computer order entry alert facilitates enhanced awareness of drug interactions that may curtail inappropriate medication use. ASP mediated PAF implemented after the computer order alert provides an opportunity to consolidate alertmediated changes in prescribing practices through cultural change allowing for sustainability and attenuating alert fatigue. Reproducing this study using a larger sample size with a longer duration of follow up will serve to test whether a reduction in PPI-antibiotic coadministration can be sustained, the independent impact of computer order alerts and ASP and whether the reduction in co-administration lowers CDI rates.

\section{Additional file}

Additional file 1: Table S1. Raw monthly data for the primary and secondary outcomes. (XLSX $11 \mathrm{~kb}$ )

\section{Abbreviations}

ASP, antibiotic stewardship program; CDI, Clostridium difficile infection; $C P O E$, computer order entry; DOT, days of therapy; PAF, prospective audit and feedback; PPI, proton pump inhibitor

\section{Acknowledgements \\ We would like to thank Gabor Kandel for his helpful and insightful comments with earlier drafts.}

\section{Funding}

A Micro-innovation Grant from the Toronto East General Hospital Department of Medicine and the University of Toronto, Faculty of Medicine supported this project. The authors independent of the supporting agencies conducted the study; collected, analyzed, and interpreted the data; and prepared, reviewed, and approved the manuscript. 


\section{Availability of data and materials}

The dataset containing the raw data supporting the conclusions of this article is included within the article as an Additional file 1: Table S1.

\section{Authors' contributions}

One author (JEP) had full access to the data in the study and takes responsibility for the integrity of the data and the accuracy of the data analysis. JM takes responsible for the integrity of the statistical analysis. JEP and SG were responsible for study design and data acquisition. JEP and SG were responsible for obtaining funding and study supervision. All authors were involved in the analysis and interpretation of the data and critical revision of the manuscript for intellectual content. All authors read and approved the final manuscript.

\section{Competing interests}

The authors declare that they have no competing interests.

\section{Consent for publication}

Not applicable.

\section{Ethics approval and consent to participate}

Ethics approval was granted from Toronto East General Hospital's Research Ethics Board.

\section{Author details}

${ }^{1}$ Department of Medicine, University of Toronto, Toronto M5S 1A8, Canada. ${ }^{2}$ Pharmacy Department, Toronto East General Hospital, Toronto M4C 3E7, Canada. ${ }^{3}$ Division of Infectious Diseases, Toronto East General Hospital, 825 Coxwell Avenue, Toronto, ON M4C 3E7, Canada. ${ }^{4}$ Department of General Internal Medicine, Toronto General Hospital, Toronto M5G 2C4, Canada.

Received: 30 October 2015 Accepted: 27 June 2016

Published online: 22 July 2016

\section{References}

1. Reid M, Keniston A, Heller JC, Miller M, Medvedev S, Albert RK. Inappropriate prescribing of proton pump inhibitors in hospitalized patients. J Hosp Med. 2012;7:421-5

2. Kelly OB, Dillane C, Patchett SE, Harewood GC, Murray FE. The inappropriate prescription of oral proton pump inhibitors in the hospital setting: a prospective cross-sectional study. Dig Dis Sci. 2015;60:2280-6.

3. McDonald EG, Milligan J, Frenette C, Lee TC. Continuous proton pump inhibitor therapy and the associated risk of recurrent Clostridium difficile infection. JAMA Intern Med. 2015;175:784-91.

4. Janarthanan S, Ditah I, Adler DG, Ehrinpreis MN. Clostridium difficile-associated diarrhea and proton pump inhibitor therapy: a meta-analysis. Am J Gastroenterol. 2012;107:1001-10.

5. Freedberg DE, Lamousé-Smith ES, Lightdale JR, Jin Z, Yang YX, Abrams JA. Use of acid suppression medication is associated with risk for $C$. difficile infection in infants and children: a population-based study. Clin Infect Dis. 2015;61(6):912-7.

6. Kwok CS, Arthur AK, Anibueze Cl, Singh S, Cavallazzi R, Loke YK. Risk of Clostridium difficile infection with acid suppressing drugs and antibiotics: meta-analysis. Am J Gastroenterol. 2012;107(7):1011-9.

7. King RN, Lager SL. Incidence of Clostridium difficile infections in patients receiving antimicrobial and acid-suppression therapy. Pharmacotherapy. 2011;31(7):642-8.

8. Slimings C, Riley TV. Antibiotics and hospital-acquired Clostridium difficile infection: update of systematic review and meta-analysis. J Antimicrob Chemother. 2014;69(4):881-91.

9. Gordon D, Young LR, Reddy S, Bergman C, Young JD. Incidence of Clostridium difficile infection in patients receiving high-risk antibiotics with or without a proton pump inhibitor. J Hosp Infect. 2016;92(2):173-7.

10. Freedberg DE, Salmasian H, Abrams JA, Green RA. Orders for intravenous proton pump inhibitors after implementation of an electronic alert. JAMA Intern Med. 2015;175:452-4.

11. Lesprit P, Duong T, Girou E, Hemery F, Brun-Buisson C. Impact of a computer-generated alert system prompting review of antibiotic use in hospitals. J Antimicrob Chemother. 2009;63(5):1058-63.
12. Dellit TH, Owens RC, McGowan JE, et al. Infectious Diseases Society of America and the Society for Healthcare Epidemiology of America guidelines for developing an institutional program to enhance antimicrobial stewardship. Clin Infect Dis. 2007:44:159-77.

13. Ontario Ministry of Health and Long-Term Care. Provincial Case Definitions for Reportable Diseases. Available at: http://www.health.gov.on.ca/en/pro/ programs/publichealth/oph_standards/docs/cdi_cd.pdf. Accessed 21 September 2015.

14. Dancer SJ, Kirkpatrick P, Corcoran DS, Christison F, Farmer D, Robertson C. Approaching zero: temporal effects of a restrictive antibiotic policy on hospital-acquired Clostridium difficile, extended-spectrum $\beta$-lactamaseproducing coliforms and meticillin-resistant Staphylococcus aureus. Int J Antimicrob Agents. 2013;41:137-42

15. Stevens V, Dumyati G, Fine LS, Fisher SG, van Wijngaarden E. Cumulative antibiotic exposures over time and the risk of Clostridium difficile infection. Clin Infect Dis. 2011;53(1):42-8.

16. Brown KA, Khanafer N, Daneman N, Fisman DN. Meta-analysis of antibiotics and the risk of community-associated Clostridium difficile infection. Antimicrob Agents Chemother. 2013;57(5):2326-32.

17. Niklasson A, Lindström L, Simrén M, Lindberg G, Björnsson E. Dyspeptic symptom development after discontinuation of a proton pump inhibitor: a double-blind placebo-controlled trial. Am J Gastroenterol. 2010;105:1531-7.

18. Ojeleye O, Avery A, Gupta $\vee$, Boyd M. The evidence for the effectiveness of safety alerts in electronic patient medication record systems at the point of pharmacy order entry: a systematic review. BMC Med Inform Decis Mak. 2013;13:69.

19. Leffler DA, Lamont JT. Clostridium difficile infection. N Engl J Med. 2015:372(16):1539-48

\section{Submit your next manuscript to BioMed Central and we will help you at every step:}

- We accept pre-submission inquiries

- Our selector tool helps you to find the most relevant journal

- We provide round the clock customer support

- Convenient online submission

- Thorough peer review

- Inclusion in PubMed and all major indexing services

- Maximum visibility for your research

Submit your manuscript at www.biomedcentral.com/submit
BioMed Central 